Joachim Liedtke

Kristianstad Universität, Kristianstad

DOI: $10.19195 / 0435-5865.141 .29$

\title{
Zur Theorie der kognitiven Verarbeitungstiefe in Korrelation mit der lexikalischen Lernleistung beim Fremdsprachenerwerb
}

\section{Interdisziplinäre Kooperation und Paradigmenwechsel}

Die in den letzten Jahrzehnten verstärkt realisierte interdisziplinäre Kooperation der Sprachlehrforschung mit der Lern- und Gedächtnispsychologie sowie der Text- und Pragmalinguistik hat neue Einsichten gefördert und bedeutende Umbrüche nach sich gezogen, welche die moderne Fremdsprachendidaktik in erheblichem Maße geprägt haben. Im Zuge dieser Entwicklung fand eine Reihe von Paradigmenwechseln statt, die sich - insbesondere auch im Hinblick auf den Lexikerwerb - skizzenartig folgendermaßen umreißen lassen:

- In den 1960er-Jahren erfolgte in der Psychologie die ,kognitive Wende“ (vgl. z.B. Neisser 1967), mit der eine Abkehr vom Behaviorismus hin zum Kognitivismus einherging und

- in den 1970er-Jahren in der Sprachlehre die ,kommunikative Wende“ initiierte (vgl. z.B. Piepho 1974), die eine Abwendung vom systematischen Vokabellernen bewirkte, da dieses als Drillmethode mit daraus resultierenden, Vokabelfriedhöfen' abgestempelt wurde, was

- in den 1980er-Jahren mit Krashens ,Input-Hypothese' zur Favorisierung von Leseaktivitäten führte (vgl. z.B. Krashen 1985, 1989) und zur Folge hatte, dass

- in den 1990er-Jahren im praktischen Fremdsprachenunterricht die intentionale und systematische Vokabelarbeit weitestgehend als veraltete Methode abgelehnt und ihr zugleich auch in empirischen Untersuchungen und theoretischen Diskussionen deutlich verminderte Aufmerksamkeit geschenkt wurde.

- Erst seit Anfang der 2000er-Jahre kam es in der Sprachlehrforschung zu einer Renaissance der wissenschaftlichen Auseinandersetzung mit dem Vokabel- 
lernen (vgl. z.B. Schmitt (2000), Read (2000), Nation (2001), Kalka (2002), Meara (2002), Neuner-Anfindsen (2005), Stork (2003), (2009), (2010), Yeh (2013), Runte (2015)), wodurch seither eine immense Anzahl innovativer Ansätze initiiert wurde, die einen erneuten Paradigmenwechsel innerhalb der fremdsprachendidaktischen Lexikarbeit markieren.

Im Rahmen dieser Neuansätze wird der Fokus vor allem auch auf die kognitiven Lernbedingungen und die daraus resultierenden Konsequenzen für das Vokabellernen in den Mittelpunkt des Interesses gerückt (vgl. Abschnitt 2 zur Referenzliteratur), wobei einerseits zwar unbestritten ist, dass die Verarbeitungstiefe des Materials eine entscheidende Rolle bei der effizienten Bewältigung der Lernaufgaben spielt, andererseits jedoch weiterer Klärungsbedarf im Hinblick darauf besteht, welche kognitiven Aktivitäten und damit korrelierenden Bedingungen die besten Voraussetzungen für das erfolgreiche Vokabellernen offerieren. Der vorliegende Beitrag greift diese Fragestellung auf, wobei das Hauptaugenmerk auf die Parameter der kognitiven Verarbeitung des lexikalischen Materials und deren mögliche Auswirkungen auf die fremdsprachliche Lexiklernleistung gerichtet wird.

\section{Kognitive Verarbeitung und lexikalische Lernleistung}

$\mathrm{Zu}$ den impulsgebenden Arbeiten, die sich im Hinblick auf die Diskussion der kognitiven Verarbeitungstiefe des Lernstoffes als besonders einflussreich erwiesen haben, zählen insbesondere der viel diskutierte Ansatz von Craik und Lockhart (1972) sowie ihre daran anknüpfenden Arbeiten (vgl. z.B. Craik/Tulving 1975, Lockhart/Craik 1990, Craik 2002). Das Hauptverdienst von Craik und Lockhart besteht vor allem darin, anstelle des bis dahin gängigen statischen Strukturmodells des Gedächtnisses - mit je einem Block für das (i) sensorische, (ii) kurzzeitige und (iii) langzeitige Erinnerungsvermögen - stattdessen ein dynamisches Prozessmodell zu postulieren, welches die kognitive Verarbeitungstiefe des Lernstoffes als zentralen Parameter der Behaltensleistung ins Zentrum der Aufmerksamkeit rückt. Craik und Lockhart unterscheiden dabei drei unterschiedliche Verarbeitungsebenen, wobei sie sowohl der physikalischen (semiotisch-orthographischen) als auch der akustischen (phonetisch-phonologischen) Ebene als sensorischem Input vornehmlich oberflächlichen Charakter zusprechen, während auf der semantischen Ebene offensichtlich die größte kognitive Verarbeitungstiefe erzielt wird, weshalb dieser Level demnach als besonders relevant für die Behaltensleistung zu bewerten ist. Craik/Lockhart verstehen ihren Entwurf als generelles Gedächtnismodell (,framework for memory research'), welches anhand von verbalem Material erläutert und unter anderem von Craik/Tulving (1975) mit Hilfe konkreter Testaufgaben empirisch überprüft wurde, wobei das Sprachmaterial allerdings ausschließlich muttersprachliche Beispiele enthält und fremdsprachliche Aspekte somit unberücksichtigt bleiben. 
Prinzipiell lässt sich das Kriterium der kognitiven Verarbeitungstiefe gleichwohl auf die Prozesse des fremdsprachlichen Lexikerwerbs übertragen, da sowohl die Form- als auch die Bedeutungsebene der zu erlernenden Vokabeln den von Craik/Lockhart konzipierten Ebenen entsprechen und somit auch hier von einer unmittelbaren Kopplung der Verarbeitungstiefe an die Behaltensleistung ausgegangen werden kann. Diese Korrelation ist in etlichen daran anknüpfenden Arbeiten erörtert worden (zur Referenzliteratur vgl. die folgenden Ausführungen). Anknüpfungspunkt bildet die ,Levels of Processing Hypothesis', nach der in Anlehnung an den Ansatz von Craik/Lockhart auch für die fremdsprachliche Lexikarbeit davon auszugehen ist, dass ,the more cognitive energy a person expends when manipulating and thinking about a word, the more likely it is that they will be able to recall and use it later" (Schmitt/McCarthy 1997: 3), womit sich für die Fremdsprachendidaktik schlussfolgern lässt: "The implications extend to pedagogy, suggesting that exercises and learning strategies which involve a deeper engagement with words should lead to higher retention than 'shallower' activities" (Schmitt/McCarthy a.a.0.).

Als kritisch erweist sich hierbei allerdings die ungenaue Definition der kognitiven Verarbeitungstiefe (,deeper engagement ${ }^{\star}$ ). Dieses Manko wurde bereits von Baddeley (1978) an dem ursprünglichen Ansatz von Craik/Lockhart moniert. Im Hinblick auf das fremdsprachliche Lexiklernen haben Laufer/Hulstijn (2001) deshalb vorgeschlagen, komplettierend von einem Konstrukt auszugehen, welches neben den kognitiven Komponenten der Lexikerschließung auch die Motivation als Lernanlass einbezieht (,motivational-cognitive construct') und damit auf den drei Hauptelementen Motivation, Suche und Evaluation (,need', ,search', , evaluation') basiert (vgl. Abb. 1). Mit diesen drei zentralen Aspekten richten Laufer/ Hulstijn die Aufmerksamkeit damit sowohl auf den Bedarf bzw. die Notwendigkeit des Erlernens von ausgewählten Vokabeln (,need'), als auch auf die Bearbeitung des lexikalischen Materials im Zuge der Bedeutungserfassung (,search') und die anschließende kontextuelle Einordnung (,evaluation'), wobei die Summe dieser drei Komponenten demnach den Grad der jeweiligen Lerner-Involviertheit (,involvement-load') und die daran gekoppelte Lernleistung widerspiegelt: „In conclusion, involvement load is defined here as the combination of the presence or absence of the involvement factors Need, Search, and Evaluation. /.../ Other factors being equal, words which are processed with higher involvement load will be retained better than words which are processed with lower involvement load" (Laufer/Hulstijn 2001: 15). 


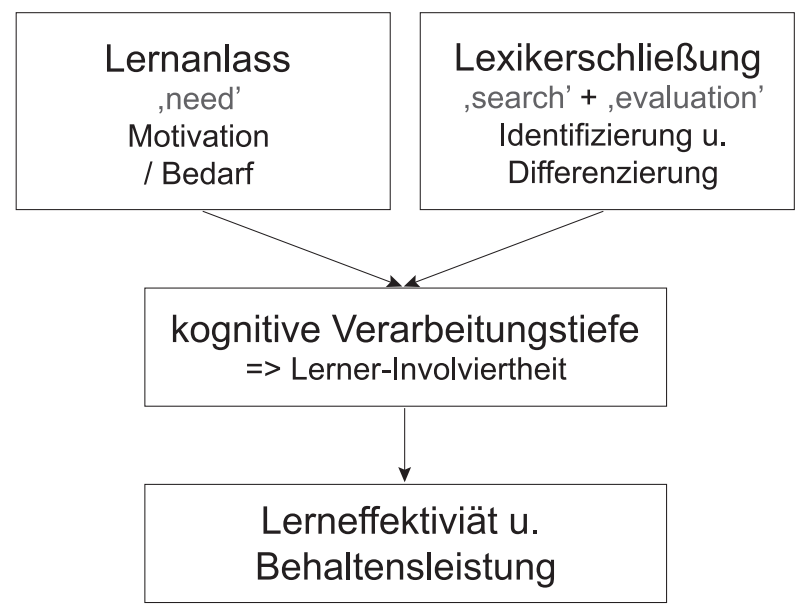

Abb. 1: Kognitiven Verarbeitungstiefe und Lerneffektivität (nach Laufer/Hulstijn 2001)

Anhand der Auswertung einer Anzahl zum Zeitpunkt ihres Entwurfs bereits vorliegender Studien verschiedener Autoren demonstrieren Laufer/Hulstijn die unterschiedlich große Wirksamkeit der jeweiligen kognitiven Aktivität der Lerner im Hinblick auf die Lerneffektivität beim fremdsprachlichen Lexikerwerb (vgl. Tab. 1).

Tab. 1: Relative Effektivität verschiedener Lexiklernmethoden (Laufer/Hulstijn 2001: 13)

\begin{tabular}{|c|c|c|}
\hline The more effective task & The less effective task & Study \\
\hline $\begin{array}{c}\text { Meaning selected from several } \\
\text { options }\end{array}$ & $\begin{array}{l}\text { Meaning explained by } \\
\text { synonym }\end{array}$ & Hulstijn 1992 \\
\hline $\begin{array}{l}\text { Meaning looked up in a } \\
\text { dictionary }\end{array}$ & $\begin{array}{l}\text { Reading with/without } \\
\text { guessing }\end{array}$ & $\begin{array}{l}\text { Knight 1994, Luppescu/Day } \\
1993\end{array}$ \\
\hline $\begin{array}{l}\text { Meaning looked up in a } \\
\text { dictionary }\end{array}$ & $\begin{array}{l}\text { Meaning provided in a } \\
\text { marginal gloss }\end{array}$ & $\begin{array}{l}\text { Hulstijn/Hollander/Greidanus } \\
1996\end{array}$ \\
\hline $\begin{array}{l}\text { Reading and a series of } \\
\text { vocabulary exercises }\end{array}$ & $\begin{array}{l}\text { Reading only } \\
\text { (and inferring meaning) }\end{array}$ & Paribakht/Wesche 1997 \\
\hline Meaning negotiated & Meaning not negotiated & Newton 1995 \\
\hline Negotiated input & Premodified input & Ellis/Tanaka/Yamazaki 1994 \\
\hline $\begin{array}{l}\text { Used in original sentences } \\
\text { (oral task) }\end{array}$ & $\begin{array}{l}\text { Used in non-original } \\
\text { sentences }\end{array}$ & Joe 1995,1998 \\
\hline $\begin{array}{c}\text { Interactionally modified } \\
\text { output }\end{array}$ & Interactionally modified input & Ellis/He 1999 \\
\hline $\begin{array}{l}\text { Used in a composition } \\
\text { (L1-L2 look-up) }\end{array}$ & $\begin{array}{l}\text { Encountered in a reading task } \\
\text { (L2-L1 look-up) }\end{array}$ & Hulstijn/Trompetter 1998 \\
\hline $\begin{array}{l}\text { Reading, words looked up in a } \\
\text { dictionary }\end{array}$ & $\begin{array}{l}\text { Reading only, words not } \\
\text { looked up }\end{array}$ & Cho/Krashen 1994 \\
\hline
\end{tabular}


Wie der Blick auf die Gegenüberstellung der verschiedenen Methoden in der Tabelle zeigt, erweisen sich demnach offensichtlich vor allem sowohl produktive Lernaufgaben als auch das rezeptiv-aktive Erschließen (,negotiation") der lexikalischen Bedeutung wie das Nachschlagen von Vokabeln im Wörterbuch sowie Leseaktivitäten, die zugleich an Lexikarbeit gekoppelt sind, als deutlich effektiver gegenüber anderen Methoden (vgl. Tab. 1). Laufer und Hulstijn sehen darin eine Bestätigung ihres Entwurfs, verstehen ihr Konzept zugleich aber ausdrücklich auch als Impulsgeber für weitere empirische und theoretische Arbeiten. $\mathrm{Zu}$ den Untersuchungen, die daran anknüpfen, zählen die Arbeiten von Hulstijn/Laufer 2001, Laufer 2005, Keating 2008, Kim 2008/2011, Martínez-Fernández 2008, Jing/Jianbin 2009, Yaqubi/Rayati/Gori 2010.

Eine erste empirische Überprüfung ihres Konzepts haben Hulstijn/Laufer (2001) bereits selbst vorgelegt. Unter Einbeziehung von fortgeschrittenen Holländisch- und Hebräisch-Lernern als Testpersonen vergleichen sie deren Lernergebnisse beim fremdsprachlichen Wortschatzerwerb sowohl unmittelbar nach der Lernaktivität als auch nach Ablauf von zwei Wochen, und zwar im Rahmen der Aufgaben (1) Leseverständnis mit Vokabelhilfe, (2) Leseverständnis plus Lückentest sowie (3) Aufsatzschreiben. Da die erste Aufgabenstellung nur einen moderaten Grad an Motivation/Bedarf und keinerlei Such- und Evaluationsaktivitäten in sich birgt, trägt sie den Involviertheitsindex $1\left(\right.$, need $^{6}: 1+$, search ${ }^{6}: 0+$, evaluation': 0 = Summe: 1). Die zweite Aufgabe beinhaltet ebenfalls einen gemäßigten Grad an Motivation/Bedarf und keine Suchaktivität, erfordert jedoch zusätzlich eine moderate Evaluation, woraus ein Index von $2(1+0+1=2)$ für die Involviertheit resultiert. Auch Aufgabe 3 erzeugt einen gemäßigten Umfang an Motivation/Bedarf sowie keinerlei Suchaktivität, allerdings erfordert sie angesichts der Vokabelaktivierung im Originalkontext eine ausgeprägte Evaluation, woraus sich nach Hulstijn/Laufer ein Involviertheitsindex von $3(1+0+2=3)$ errechnen lässt.

Die nach Abschluss der Tests ausgewerteten Ergebnisse belegen weitestgehend, dass Vokabelaktivitäten mit einem höheren Grad der Involviertheit offensichtlich zu einer besseren Behaltensleistung führen und Hulstijn/Laufer interpretieren diese empirischen Belege als weitere Bestätigung ihrer Involviertheitshypothese. Einen besonderen Vorteil des Involviertheitskriteriums sehen sie in der damit bereitgestellten Operationalisierbarkeit der kognitiven Verarbeitungstiefe, die weitere empirische Überprüfungen ermöglicht: „Because the construct of involvement can be operationalized and investigated empirically, researchers could devise tasks with different involvement loads and compare them with regard to their effect on incidental vocabulary learning" (Hulstijn/Laufer 2001: 552).

Keating (2008) knüpft daran an und untersucht ergänzend, ob sich die Involviertheitshypothese auch verifizieren lässt, wenn (1) sich die Lerner noch auf dem Anfängerniveau befinden, (2) zwischen aktiven und passiven Vokabelkenntnissen differenziert wird und (3) der zeitliche Bearbeitungsaufwand der 
Lernaufgaben ebenfalls mit in Betracht gezogen wird. Nach Auswertung der Untersuchungsergebnisse resümiert Keating: „The present study extends empirical support for the construct of task-induced involvement in L2 vocabulary learning by demonstrating that low-proficiency learners benefit from more involving tasks, that tasks with higher involvement load lead to greater gains both passive and active word knowledge, and that time on task is an important factor to consider when evaluating the relative effectiveness of vocabulary learning tasks" (Keating 2008: 382). Damit zeichnet sich ab, dass sich mit Hilfe der Involviertheitshypothese auch eine größere Anzahl von Randbedingungen des Vokabellernens im Hinblick auf ihre Bedeutung für die Lerneffektivität untersuchen lässt (wobei dabei allerdings nicht - wie teilweise in Keatings Arbeit - eine Liste mit Nonsenswörtern, sondern stattdessen authentisches Originalmaterial zugrundegelegt werden sollte, da davon auszugehen ist, dass die Verwendung von unsinnigem Material die Komponente der Motivation erheblich beeinträchtigen kann).

Kim (2008/2011) setzt die Analyse zusätzlicher Parameter fort und beschreibt die Resultate zweier Tests, die zeigen, dass sich die Involviertheitshypothese auch im Hinblick auf zwei weitere spezielle Bedingungen verifizieren lässt, nämlich mit Bezug auf (1) das Fremdsprachenniveau der Lerner (Undergrades vs. Intensive English Program, vgl. Experiment 1 sowie (2) die Aufgabenstellung in Form produktiver Übungen (Aufsatz- vs. Satzproduktion, vgl. Experiment 2, und zwar gleichermaßen sowohl bei der Kontrolle der lexikalischen Behaltensleistung unmittelbar nach dem Lernaktivität als auch bei einer zwei Wochen später durchgeführten Nachprüfung.

Auch Jing/Jianbin (2009) konzentrieren sich in ihrer Untersuchung auf die möglichen Einflüsse der Involviertheitshypothese auf den Lexikerwerb. Sie richten das Hauptaugenmerk dabei auf die rezeptiven Bedingungen im Rahmen des Hörverständnisses. Zu diesem Zweck vergleichen sie den Grad der Involviertheit in Zusammenhang mit unterschiedlich konzipierten Hörverständnisfragen, nämlich mit (1) beigefügten, doch für die Fragen irrelevanten Vokabelangaben, (2) beigefügten und für die Fragen relevanten Vokabelangaben sowie (3) beigefügten und sowohl für die Fragen als auch eine anschließende schriftliche Zusammenfassung relevanten Vokabelangaben. Auch diese Tests belegen signifikante Unterschiede des lexikalischen Lernresultate, die sich als Verifikation der Involviertheitshypothese interpretieren lassen.

$\mathrm{Zu}$ den weiteren Ansätzen, die ebenfalls eine empirische Überprüfung und Präzisierung der Involviertheitshypothese anstreben, zählt auch die Untersuchung von Martínez-Fernández (2008). Sie widmet ihre Arbeit ebenfalls den rezeptiven Bedingungen und konzentriert sich dabei auf Leseaktivitäten mit unterschiedlich konzipierten Zusatzaufgaben: (1) Leseaufgabe mit Vokabelhilfe (L1-Übersetzungen), (2) Leseaufgabe kombiniert mit Lückentest (Einsetzen der fehlenden Ausdrücke in den Textzusammenhang) und (3) Leseaufgabe kombi- 
niert mit bereitgestellten Vokabelübersetzungen in Multiple-Choice-Form. Anschließend erfolgt eine Überprüfung der lexikalischen Lernerfolge in vier Tests und der kontrastiven Gegenüberstellung von Form/Inhalt sowie Produktion/Rezeption: (1) L2-Wortform-Produktion (von der L1-Übersetzung ausgehend), (2) L2-Wortbedeutungs-Produktion (unter Vorgabe eines L2-Satzes einschließlich der L1-Übersetzung), (3) L2-Wortform-Rezeption (durch Auswahl des Zielwortes aus drei Alternativen) und (4) L2-Wortbedeutungs-Rezeption (durch Auswahl des korrekten Zielwortes aus drei im vorausgegangenen Multiple-Choice-Test bereits vorgelegten Alternativen). Komplettiert werden diese Daten durch die Gegenüberstellung von konkreten und abstrakten Bedeutungen des Lernwortschatzes sowie Gedankenprotokolle der Probanden, um den Grad der Bewusstmachung (,awareness') mit zu erfassen.

Die Ergebnisse zeigen, dass unterschiedliche Grade der Involviertheit demnach zu unterschiedlichen Ebenen der Bewusstmachung führen, allerdings abweichend von der als Ausgangspunkt gewählten Involviertheitshypothese: „Findings in this study show that tasks with different degrees of involvement load induced different levels of awareness, although not in the direction predicted by the Involvement Load Hypothesis.“ (Martínez-Fernández 2008: 225). Martínez-Fernández schlussfolgert daraus, dass sich offensichtlich Aufgabenstellungen mit vorrangig produktiven Übungsanteilen durch eine besonders hohe Relevanz für die lexikalische Lernleistung auszeichnen, wobei der Einfluss so dominant sich kann, dass die Komponente der Evaluation davon überlagert wird, was eine erforderliche Präzisierung der Involviertheitshypothese nahelegt: ,since the highest involvement load was confounded with output-orientation, it seems likely that output, and not evaluation, had the highest effect on vocabulary development in those studies" (ebd.: 227).

Auch Yaquiv/Rayati/Gorgi (2010) schlussfolgern nach Abschluss ihrer empirischen Untersuchungen, dass eine Komplettierung der Involviertheitshypothese vorzunehmen sei. Sie konzentrieren sich in ihrer Arbeit auf die entscheidende Frage, inwieweit andere Einflussfaktoren wie die bereits oben erwähnten Output-Aktivitäten die Relevanz der Komponenten ,need', ,search, und ,evaluation' überlagern können und damit eine Erweiterung der Involviertheitshypothese erforderlich machen. Im Zentrum ihrer Überprüfung steht die Relevanz des Aufgabentyps in Kombination mit dem Leseverständnis und der damit verbundenen Lerneraktivitäten. Dabei vergleichen sie die lexikalischen Lernfortschritte im Rahmen dreier Aufgaben: (1) dem Leseverständnis unter Heranziehung eines Wörterbuchs, (2) dem Leseverständnis mit kombiniertem Lückentest und beigefügtem Glossar der entsprechenden Vokabeln sowie (3) dem Leseverständnis mit anschließender Essay-Erstellung zum Thema, wobei die relevanten Vokabeln ebenfalls in Form einer zweisprachigen Liste bereitgestellt wurden. 
Die anschließend vorgenommene Überprüfung der lexikalischen Behaltensleistung (von 10 Testwörtern) bestätigt die signifikant höhere Lernleistung im Zuge der produktiven Aufgabe (Leseaktivität plus Essay-Erstellung) gegenüber der rezeptiven Übung (Leseaktivität plus Wörterbuch-Nutzung) und konsolidiert damit die Hypothese im Hinblick auf den Aufgabentyp: „This indicates that, everything else being equal, output-oriented tasks are far more conductive to learning vocabulary than input-oriented tasks" (Yaqubi/Rayati/Allemzade Gorgi 2010: 159). Dagegen resultiert aus der Leseübung mit Wörterbuch (Experiment 1) trotz des angesetzten höheren Involviertheitsindexes und entgegen der Erwartung der Autoren eine deutlich geringere Behaltensleistung als bei der Leseübung inklusive Lückentext mit niedrigerem Index (Experiment 2). Die Autoren schlussfolgern daraus, dass die Operationalisierung der Verarbeitungstiefe deshalb einer weiteren Präzisierung bedarf, wobei das Augenmerk insbesondere auch auf den Aufgabentyp zu richten ist: „the study suggests that the grading of components of involvement load hypothesis should be further fine grained to be proportionate to the depth of processing (in case of this study, evaluation") that a task demands. /.../ That is, the processing load brought to bear by task type may well affect word retention, a point needing further empirical studies" (ebd.: $160 \mathrm{f}$.).

\section{Konstruktive Kritik: Komplexität und Korrelationen der kognitiven Verarbeitung}

Zusammenfassend lässt sich damit konstatieren, dass der von Craik/Lockhart (1972) entworfene und später von Hulstijn/Laufer (2001) für den fremdsprachlichen Lexikerwerb adaptierte Ansatz der kognitiven Verarbeitungstiefe und der daraus resultierenden Einflüsse auf die Lerneffektivität mit Hilfe verschiedener daran anknüpfender empirischer Untersuchungen als Grundkonzept weitestgehend verifiziert werden konnte. Zugleich zeichnet sich aber auch die notwendige Erweiterung dieses Ansatzes ab, um so der Komplexität und Korrelationen der kognitiven Parameter angemessen gerecht werden zu können. Dieser Erweiterungsbedarf betrifft sowohl die bereits in der Ursprungsversion enthaltenen Komponenten der Motivation (,need $)$, Suche (, search') und Bewertung (,evaluation') als auch zusätzliche Faktoren, die damit unmittelbar oder mittelbar verknüpft sind.

Im Hinblick auf das Gesamtkonzept sind drei Hauptkomplexe zu integrieren: (I) die Charakteristika des jeweiligen Lernindividuums, (II) die Konditionen des ablaufenden Lernprozesses sowie (III) die Eigenschaften des lexikalischen Lernstoffes (vgl. Abb. 2).

Die drei Komponenen (,need', ,search', ,evaluation') der Involviertheitshypothese von Laufer/Hulstijn sind darin eingebettet und zugleich mit direkt oder indirekt korrelierenden Komponenten verbunden. Auf diese Weise erfolgt eine 


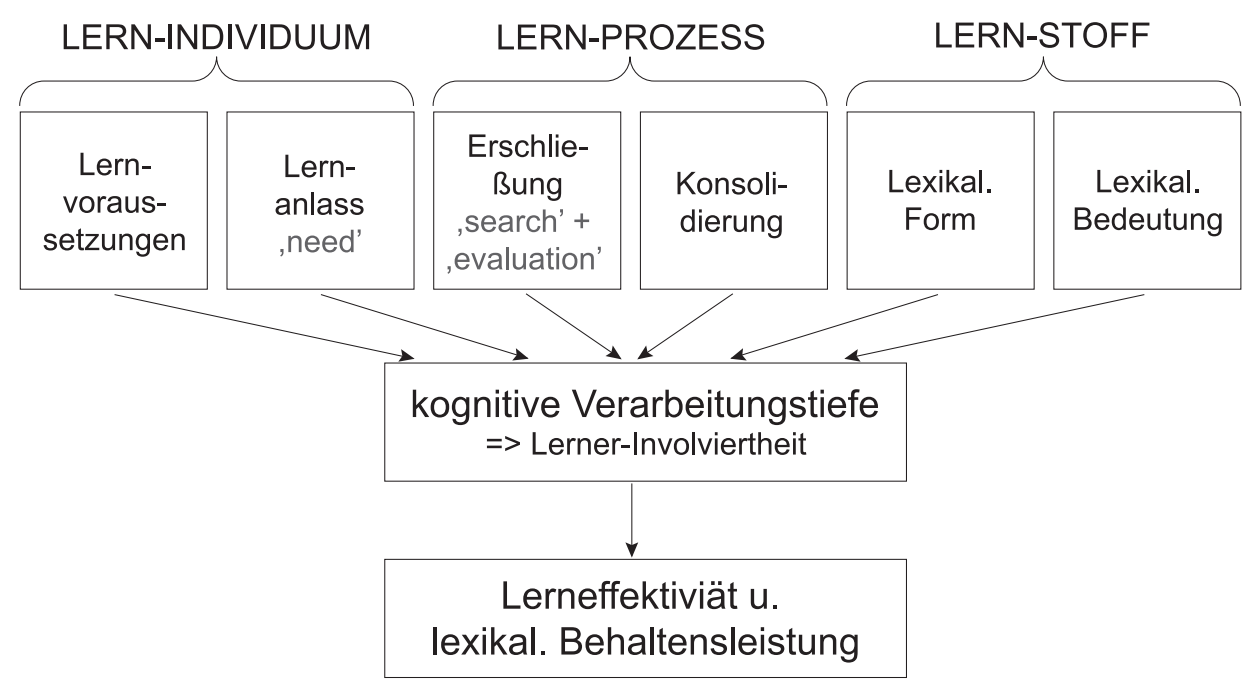

Abb. 2: Erweitertes Konzept der kognitiven Verarbeitungstiefe und Lerneffektivität

Verknüpfung von Lernmotivation/-anlass (,need') und Lernvoraussetzungen des Lernindividuums, von Erschließung (,search'+,evaluation') und Konsolidierung im Zuge des Lernprozesses sowie von Form und Bedeutung der zu erlernenden Vokabeln, um sowohl die Korrelationen dieser Parameter als auch ihre Relevanz für die kognitive Verarbeitungstiefe und den daraus resultierenden Lernerfolg einbeziehen zu können. Zusätzlich lassen sich auf diesem Wege weitere Differenzierungen innerhalb der genannten Hauptkomplexe realisieren, um deren spezifische Einflüsse auf die kognitive Verarbeitungstiefe zu analysieren (vgl. Abb. 3).

So ist die Motivation (,need') ergänzend dahingehend zu differenzieren, auf welchem Ursprung sie basiert: Ist sie selbst- oder fremdbestimmt und mit welchen Anteilen beruht dies auf einer Notwendigkeit (z.B. Sprachprüfung, Auslandsaufenthalt), der Relevanz und Interessantheit der Lerninhalte und einer möglichen emotionalen Einbindung (infolge persönlicher Kontakte u. Beziehungen)?

Innerhalb des Komplexes der Lernprozesse und Lernprogression ist neben der bereits berücksichtigten qualitativen Erschließung (,search') und Differenzierung (,evaluation') des lexikalischen Lernmaterials insbesondere eine Komponente der Konsolidierung zu komplettieren, um somit auch die quantitativen Kriterien der Aneignung und Festigung der Vokabelkenntnisse durch Zeitaufwand und Häufigkeit beim Erlernen und zeitverzögerten Wiederholen zu erfassen, wobei auch der Aufgabentyp und dessen Übungsanteile für die verschiedenen kommunikativen Fertigkeiten (mündlich/schriftlich u. rezeptiv/produktiv) einzubeziehen sind.

Ein dritter Bereich betrifft die Komplexität des lexikalischen Lernmaterials. Dieser Aspekt wird in der ursprünglichen Fassung der Involviertheitshypothese völlig ausgeklammert, obgleich mit Gewissheit davon auszugehen ist, dass sowohl der Schwierigkeitsgrad als auch der Interessantheitsgrad des Lernstoffes nicht nur 


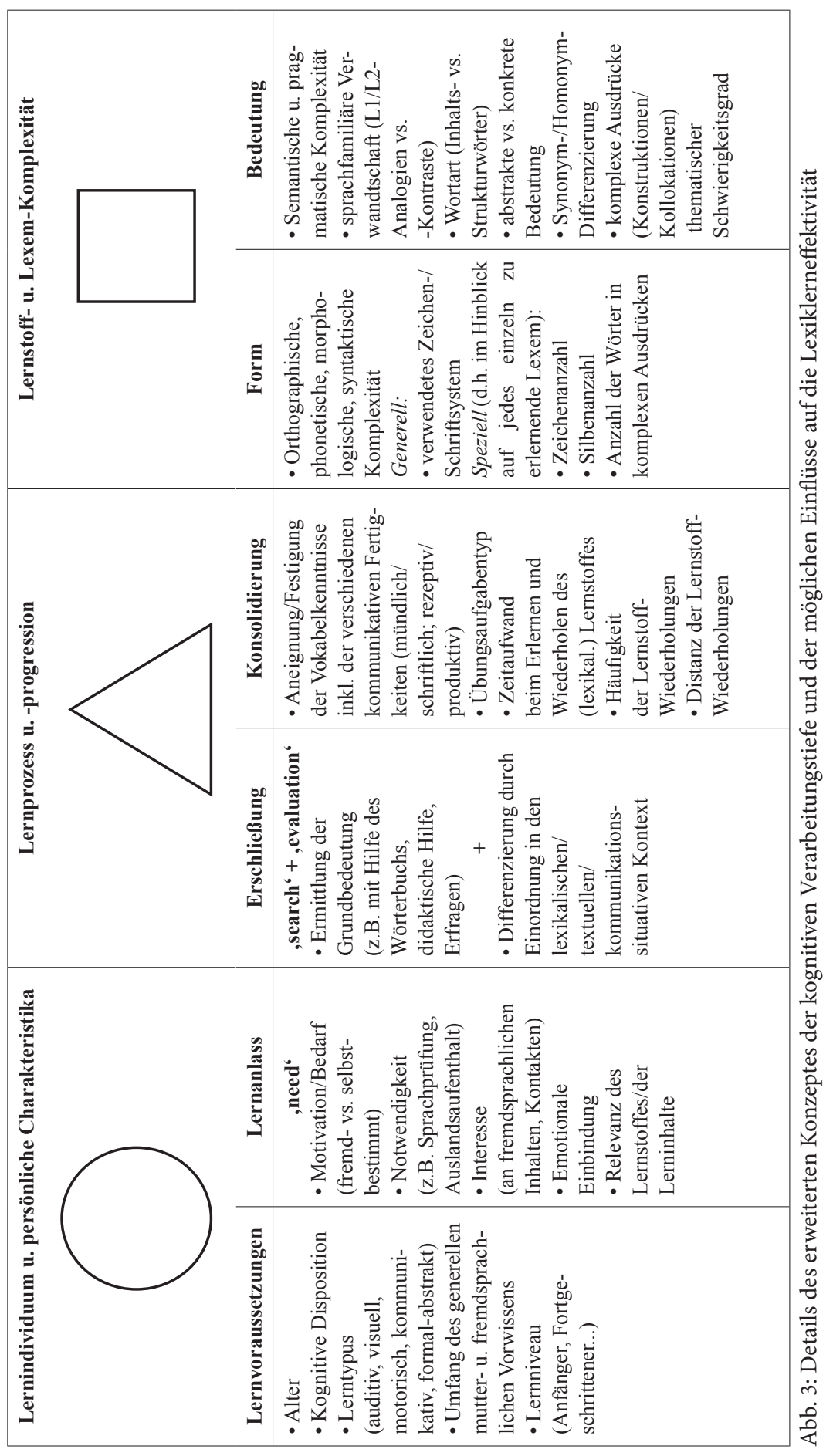


die Lernprogression, sondern auch die Lernmotivation und die Lerneffektivität erheblich prägen können. Dies gilt umso mehr, da jedem einzelnen zu erlernenden lexikalischen Ausdruck ein eigener Grad der formalen (orthographisch-fonetischen) und inhaltlichen (semantisch-pragmatischen) Komplexität zuzuschreiben ist.

Ein weiterer, die Lerner-Involviertheit in großem Maße prägender Aspekt betrifft die innerhalb dieses komplexen Gefüges existierenden Korrelationen, da nicht nur zentrale Relationen zwischen den Hauptkomponenten Lernindividuum,

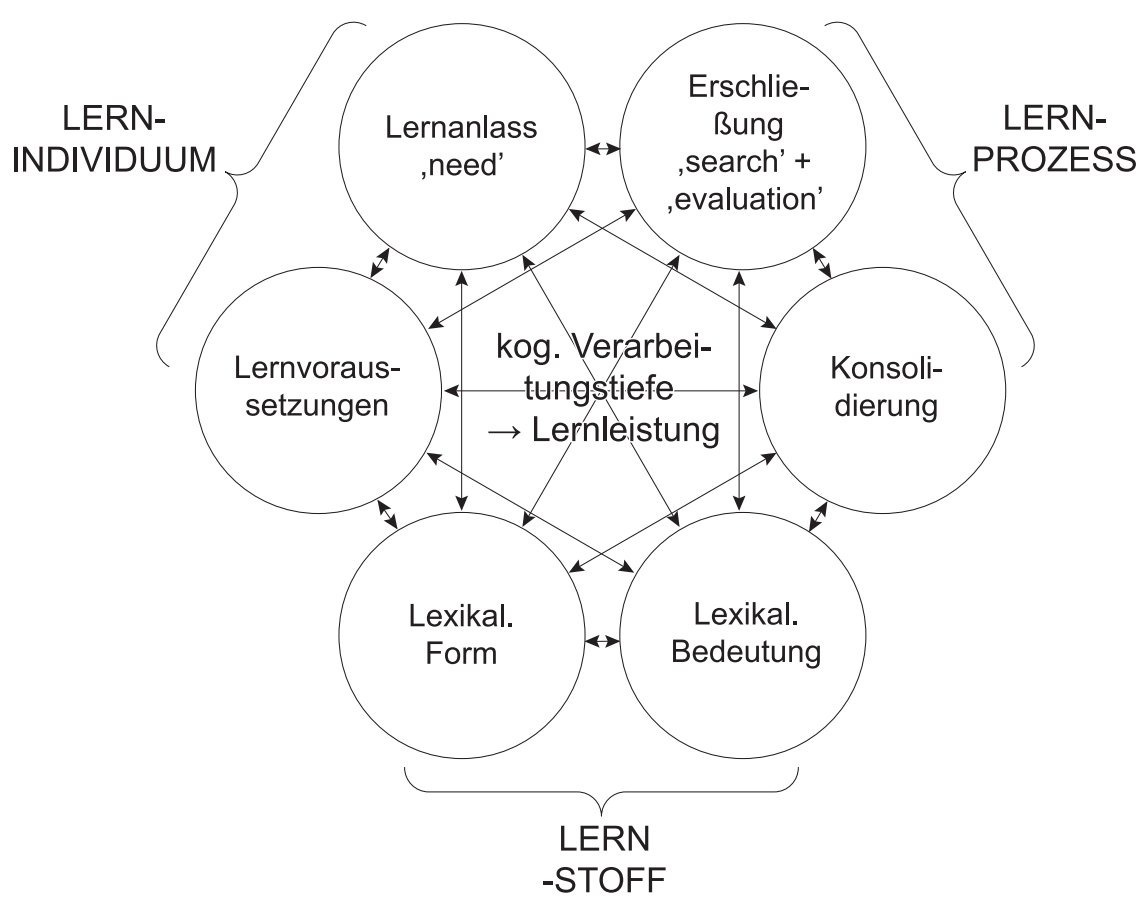

Abb. 4: Komplexe korrelative Verknüpfung von kognitiver Verarbeitungstiefe u. Lernleistung

Lernprozess und Lernstoff bestehen, sondern zusätzlich von etlichen Querverbindungen zwischen den übrigen Teilkomponenten auszugehen ist (vgl. Abb. 4). Deren vielfältige direkte und indirekte Beziehungen erschweren erheblich die Definition abhängiger und unabhängiger Variablen empirischer Analysen, womit ersichtlich ist, dass eine adäquate systematische Erfassung aller am Lexikerwerb beteiligten Parameter nur eingelöst werden kann, wenn es gelingt, ein geeignetes Analyse-Instrumentarium bereitzustellen.

Als Lösungsansatz kommt hier in erster Linie eine gezielte Nutzung der computergestützten Spracherwerbsanalyse in Frage (vgl. hierzu Liedtke (2010) mit einem detaillierten Entwurf sowie Schmitt (2010: 97 ff.) mit einem resümierenden Übersicht zum aktuellen Stand von ,computer simulations of vocabulary“). Erst wenn unter Einsatz geeigneter Technologien neue Wege beschritten werden, um 
der immensen Komplexität der am Fremdsprachenerwerb beteiligten Faktoren mit Hilfe eines computerunterstützten Konzepts einer systematischen und synthetischen Analyse zugänglich zu machen, kann es gelingen, den komplexen korrelativen Verknüpfungen zwischen der kognitiven Verarbeitung und der sie beeinflussenden Parameter angemessen gerecht zu werden.

\section{Literaturverzeichnis}

Baddeley, Alan D.: The trouble with levels: a reexamination of Craik and Lockhart's Framework for memory research. In: Psychological Review 85, 3, Mai 1978, S. 139-152.

Cho, Kyung-Sook / Stephen D. Krashen: Acquisition of vocabulary from the Sweet Valley Kids series: Adult ESL acquisition. In: Journal of Reading 37, 8, Mai 1994, S. 662-667.

Craik, Fergus I.M.: Levels of processing: Past, present ... and future? In: Memory 10, 5/6, 2002, 305-318.

Craik, Fergus I.M. / Endel Tulving: Depth of processing and the retention of words in episodic memory. In: Journal of Experimental Psychology: General 104, 3, 1975, S. 268-294.

Craik, Fergus I.M. / Robert S. Lockhart: Levels of processing: A framework for memory research. In: Journal of Verbal Learning and Verbal Behavior 11, 6, Dec 1972, S. 671-684.

Ellis, Rod / Xien He: The roles of modified input and output in the incidental acquisition of word meanings. In: Studies in Second Language Acquisition 21, 1999, S. 285-301.

Ellis, Rod / Yoshihiro Tanaka / Asako Yamazaki: Classroom interaction, comprehension, and the acquisition of L2 word meanings. In: Language Learning 44, 3, Sept. 1994, S. 449-491.

Hulstijn, Jan H.: Retention of inferred and given word meanings: Experiments in incidental vocabulary learing. In: Anaud, Pierre, J. / Béjoint, Henri (Hrsg.): Vocabulary and Applied Linguistics. London 1992, S. 113-125.

Hulstijn, Jan H. / Merel Hollander, M. / Tine Greidanus: Incidental vocabulary learning by advanced foreign-language students: The influence of marginal glosses, dictionary use, and reoccurrence of unknown words. In: The Modern Language Journal 80, 1996, S. 327-339.

Hulstijn, Jan H. / Pascale Trompetter: Incidental learning of second language vocabulary in computer-assisted reading and writing tasks. In: Albrechtsen Dort / Hendricksen Birgit/ Mees, Inger/ Poulsen Erik (Hrsg.): Perspectives on Foreign and Second language Pedagogy. Odense 1998, S. 191-200.

Jing, Lu / Huang Jianbin: An empirical study of the involvement load hypothesis in incidental vocabulary acquisition in EFL listening. In: Polyglossia 16, Feb. 2009, S. 1-10.

Joe, Angela: Text-based tasks and incidental vocabulary learning: A case study. In: Second Language Research 11, 1995, S. 149-158.

Joe, Angela: What effects do text-based tasks promoting generation have on incidental vocabulary acquisition? In: Applied Linguistics 19, 3, 1998, S. 157-377.

Kalka, Katarzyna: Effizienz verschiedener Lehrmethoden zur Wortschatzvermittlung. Marburg 2002.

Keating, Gregory D.: Task effectiveness and word learning in a second language: The involvement load hypothesis on trial. In: Language Teaching Research 12, 3, Jul. 2008, S. 365-386.

Kim, YouJin: The role of task-induced involvement and learner proficiency in L2 vocabulary acquisition. In: Language Learning 58, 2, 285-325. Nachdruck: Language Learning 61: Suppl. 1, June 2011, 100-140.

Knight, Susan: Dictionary: the tool of last resort in foreign language reading? A new perspective. In: Modern Language Journal 78, 1994, S. 285-299.

Krashen, Stephen D.: The Input Hypotheses: Issues and Implications. New York 1985.

Germanica Wratislaviensia 140, 2016

(C) for this edition by CNS 
Krashen, Stephen D.: We acquire vocabulary and spelling by reading: additional evidence for the input hypothesis. In: Modern Language Journal 73, 4, 1989, S. 440-464.

Laufer, Batia: Focus on form in second language vocabulary learning. In: EUROSLA Yearbook 5. 2005, S. 223-250.

Laufer, Batia / Jan Hulstijn: Incidental vocabulary acquisition in a second language: the construct of task-induced involvement. In: Applied Linguistics 22, 2001, S. 1-26.

Liedtke, Joachim: Sprachenlernen und Individualität: Zur Synthese von intentionalem und inzidentellem sowie kontextfreiem und kontextualisiertem Lexiklernen im Rahmen einer interdisziplinären Fremdsprachendidaktik. In: Stroh, Cornelia (Hrsg.): Von Katastrophen, Zeichen und vom Ursprung der menschlichen Sprache. Bochum 2010, S. 125-155.

Lockhart, Robert S. / Fergus I.M. Craik: Levels of Processing: a retrospective commentary on a framework for memory research. In: Canadian Journal of Psychology 44, 1, 1990, S. 87-112.

Luppescu, Stuart / Richard R. Day: Reading, dictionaries, and vocabulary learning. In: Language Learning 43, 2, 1993, S. 263-287.

Martínez-Fernández, Ana: Revisiting the involvement-load hypothesis: Awareness, type of task and type of item. In: Melissa Bowles / Rebecca Foote / Silvia Perpiñán (Hrsg.): SLRF 2007: Selected Proceedings volume, Cascadilla Proceedings Project, Somerville, MA., 2008, S. 201-228.

Meara, Paul: Review article. The rediscovery of vocabulary. In: Second Language Research 18, 4, 2002, S. 393-407.

Nation, I.S. Paul: Learning vocabulary in another language. Cambridge 2001.

Neuner-Anfindsen, Stefanie: Fremdsprachenlernen und Lernerautonomie. Sprachlernbewusstsein, Lernprozessorganisation und Lernstrategien zum Wortschatzlernen in Deutsch als Fremdsprache. Baltmannsweiler 2005.

Neisser, Ulrich: Cognitive psychology. New York, NY 1967.

Newton, Jonathan: Task-based interaction and incidental vocabulary learning: A case study. In: Second Language Research 11, 2, 1995, S. 159-177.

Paribakht, T. Sima / Marjoric Wesche: Vocabulary enhancement activities and reading for meaning in second language vocabulary acquisition. In: J. Coady / T. Huckin (Hrsg.): Second Language Vocabulary Acquisition. A Rationale for Pedagogy. Cambridge 1997, S. 174-200.

Piepho, Hans-Eberhard: Kommunikative Kompetenz als übergeordnetes Lernziel im Englischunterricht. Limburg 1974.

Read, John: Assessing Vocabulary. Cambridge: Cambridge 2000.

Runte, Maren: Lernerlexikographie und Wortschatzerwerb (Lexicographica. Series Maior). Frankfurt am Main 2015.

Schmitt, Norbert: Vocabulary in Language Teaching. Cambridge 2000.

Schmitt, Norbert: Researching Vocabulary. A Vocabulary Research Manual. Hampshire, New York, NY 2010.

Schmitt, Norbert / Michael McCarthy: Vocabulary: Description, acquisition and pedagogy. Cambridge 1997.

Stork, Antje: Vokabellernen: Eine Untersuchung zur Effizienz von Vokabellernstrategien (Giessener Beiträge zur Fremdsprachendidaktik). Giessen 2003.

Stork, Antje: Vokabellernen im DaZ-Unterricht: erproben, reflektieren, sich austauschen. In: Deutsch als Zweitsprache 3, 2009, S. 22-28.

Stork, Antje: Wortschatzerwerb. In: Hallet, Wolfgang / Königs, Frank G. (Hrsg.): Handbuch Fremdsprachendidaktik. Kallmeyer 2010. S. 104-107.

Yaqubi, Baqer / R.A. Rayati / N. Allemzade Gorgi: The involvement load hypothesis and vocabulary learning: The efffect of task types and involvement index on L2 vocabulary acquisition. In: The Journal of Teaching Language Skills 2, 1, Spring 2010, S. 145-163.

Yeh, Lien Chuan: Gezielte Vermittlung und Akzeptanz von Wortschatzlernmethoden: Eine empirische Untersuchung über den Einfluss von Lehrmethoden auf das Lernverhalten bei DaF-Studierenden in Taiwan und in China. Tectum, 2013. 


\begin{abstract}
Der Rückblick auf die Entwicklung der Fremdsprachenerwerbsforschung während der letzten Dezennien belegt eine wachsende interdisziplinäre Kooperation insbesondere zwischen der Sprachdidaktik und der Lernpsychologie. Der vorliegende Text reflektiert diese Fortschritte unter besonderer Berücksichtigung der Involvement-Load Hypothese von Laufer/Hulstijn (2001) inklusive daran anknüpfender Arbeiten. Nach der Diskussion der Vor- und Nachteile des Originalkonzeptes und seiner nachfolgenden Erweiterungen werden Vorschläge für mögliche Komplettierungen unterbreitet, um den komplexen Korrelationen des Fremdsprachenerwerbs und seiner theoretischen Implikationen gerecht werden zu können.
\end{abstract}

Schlüsselwörter: Fremdsprachenerwerb, Lexik, Verarbeitungstiefe, Involvement-Load Hypothese

\title{
On the theory of the levels of processing in correlation with foreign language vocabulary learning
}

This paper briefly reviews the changes of theoretical paradigms in foreign language learning research during the last five decades. As a result of these changes, vocabulary acquisition theory has become more and more influenced by interdisciplinary cooperation, especially by psychological concepts dealing with word learning and retention. This article focuses on the involvement-load hypothesis, created by Laufer/Hulstijn (2001) and completed by other researchers during the last ten years. The advantages and disadvantages of the original concept and its later derivatives are discussed. Necessary enlargements are also suggested in order to account for the complex correlations that are involved in the process of vocabulary acquisition and its theory.

Keywords: vocabulary learning, levels of processing, involvement-load hypothesis

Joahim Liedtke

Kristianstad University

SE-291 88 Kristianstad

Sweden

E-Mail: Joachim.Liedtke@hkr.se 